\title{
Microcomputer Interface for Computer-Controlled Enzyme Kinetic Studies with the Monolayer Technique
}

\author{
Gabrielle M. DONNE-OP DEN Kelder, HaRry VAN DER WILDT, \\ AND GERARD H. DE HAAS \\ Laboratory of Biochemistry, State University of Utrecht, Transitorium 3, \\ Padualaan 8, NL-3584 CH Utrecht, The Netherlands
}

Received November 10, 1983

\begin{abstract}
A microcomputer interface for computer-assisted monolayer experiments was developed, tested, and used for analysis of the enzymatic hydrolysis by pancreatic phospholipases $A_{2}$ (EC 3.1.1.4) of 1,2-didodecanoyl-sn-glycero-3-sulfate monitored under constant surface pressure. The interface described multiplexes two different analog signals onto one set of 12 data input lines to the computer. One signal is obtained from an electromicrobalance which measures surface pressure of a monomolecular substrate layer in a two-compartment trough. The second signal comes from a 10-turns precision potentiometer which measures the position of a surface barrier. Both signals together determine a velocity value which is output via four data lines to a DC motor that drives the barrier. Software developed specifically to drive the interface, to store data, and to keep pressure constant is described. (1) 1984 Academic Press, Inc.

KEY WORDS: phospholipase $A_{2}$; negatively charged substrates; monolayer technique; computercontrolled system; interface; software.
\end{abstract}

The ability to acquire monolayer data on line and to analyze the data very shortly thereafter provides the user with an accurate and fast tool to determine velocities and corresponding lag times of phospholipases acting on monomolecular lipid films. With this computer-interfaced system the tedious analysis of the data by hand calculation is no longer needed. The interface was built at relatively low cost (approx \$500) and can be used in combination with any 8-bit microprocessor.

The computer-controlled monolayer device which is discussed in this paper was used to gain insight into the interfacial kinetics of pancreatic PLAs ${ }^{1}$ on negatively charged sub-

\footnotetext{
${ }^{1}$ Abbreviations used: PLA, phospholipase $\mathrm{A}_{2}$; di- $\mathrm{C}_{12} \mathrm{GS}$, 1,2-didodecanoyl-sn-glycero-3-sulfate; di- $\mathrm{C}_{10} \mathrm{PC}, 1,2$-didecanoyl - $s n$ - glycero - 3 - phosphocholinc; di - $\mathrm{C}_{8} \mathrm{PC}, 1,2$ dioctanoyl-sn-glycero-3-phosphocholine; iso-PLA, isoenzyme of porcine PLA; LSB, least significant bit; DAS, data-acquisition system; PTM, programmable timer module; ADC, analog to digital converter; PIA, peripheral interface adaptor, Tris, tris(hydroxymethyl)aminomethane.
}

strates. Although most previous studies have been done on the neutral diacyl-sn-glycero-3phosphocholines, there were several reasons for including negatively charged lipids as well. The enzyme acts in the intestine on bile saltstabilized phospholipid micelles. These conjugated bile salts confer a strong negative charge on the phospholipid dispersion. Also in the absence of bile salts the pancreatic enzymes are known to attack preferentially anionic phospholipids $(1,2)$.

\section{EXPERIMENTAL}

Enzymes. The pancreatic pro- and isoprophospholipases $\mathrm{A}_{2}$ from pig, ox, sheep, horse, and man were purified from pancreatic tissue and converted into phospholipases $\mathrm{A}_{2}$ by limited proteolysis as described by Nieuwenhuizen et al. (3), Fleer et al. (4), Dutilh et al. (5), Evenberg et al. (b), and Grataroli et al. $(7,8)$, respectively. Pure bee venom phospholipase was a gift of Professor C. A. Vernon 
and was purified according to Shipolini $e t$ al. (9).

Protein concentrations were calculated from the absorbance at $280 \mathrm{~nm}$ by using $E_{1}^{1 \%} \mathrm{~cm}$ values of $\mathbf{1 2 . 3}$ for bovine and equine PLA. For porcine PLA (and iso-PLA), human PLA, and sheep PLA a value of 13.0 was used.

Film constituent. The medium-chain raceme 1,2-didodecanoyl-sn-glycero-3-sulfate was prepared as described by Hille (10). The L-compound was isolated from the racemic mixture by treatment with phospholipase $\mathrm{A}_{2}$ and reacylation of the lyso compound (11). The short-chain 1,2-didecanoyl-sn-glycero-3phosphocholine was prepared as described by Cubero Robles and van den Berg (12).

Aqueous subphase. Deionized-distilled water was used in all experiments. In all assays the aqueous subphase was composed of a 10 $\mathrm{mM}$ Tris buffer adjusted to $\mathrm{pH} 8$ in $100 \mathrm{mM}$ $\mathrm{NaCl}$ and $20 \mathrm{mM} \mathrm{CaCl}_{2}$. Absolute surface ten- sion values and the absence of surface tension changes upon compression indicated that no surface active impurities were present after cleaning the surface several times with a Pasteur pipet connected to a vacuum pump.

Monolayer experiments. All kinetic experiments were performed with the "zero-order trough" (13) containing two compartments connected by a small surface channel (see Fig. 1). Enzyme $(1-10 \mu \mathrm{g})$ was injected under the film in the left compartment only, whereas the substrate film covers both compartments. The right compartment, containing a mobile barrier, was used to correct for substrate molecules removed from the film by enzymatic hydrolysis, with the surface pressure kept constant. The latter is measured with a Wilhelmy plate attached to a microbalance, which is in turn connected to a microprocessor that controls the movement of the mobile barrier. In the left, stirred compartment temperature was

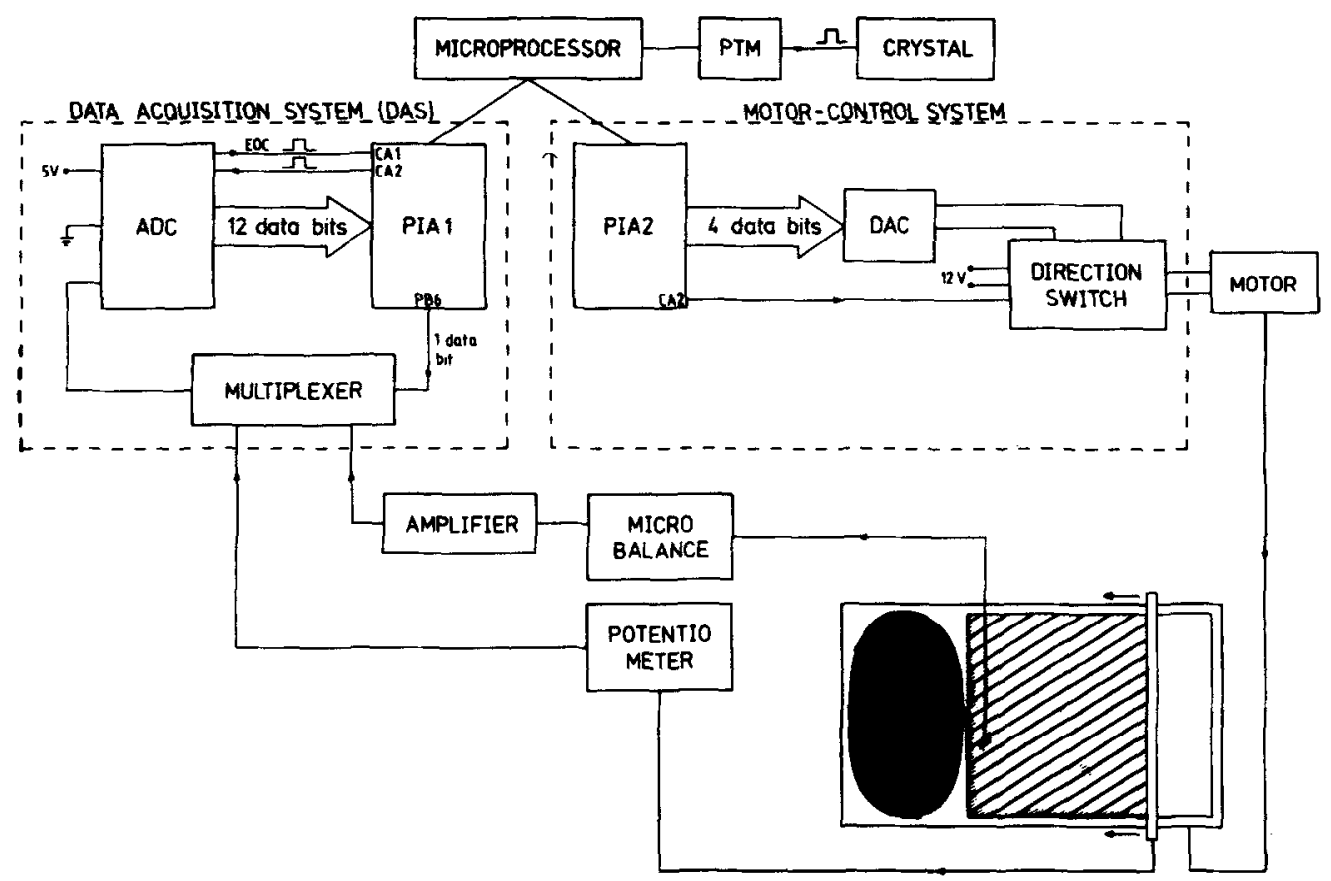

FIG. 1. Schematic representation of the interface design connecting microprocessor and peripheral devices. PIA1 and PIA2, peripheral interface adapters, are 16-bit I/O (input/output) programmable devices; ADC, analog to digital converter; DAC, digital to analog converter; EOC, end of conversion; CA1 and CA2, programmable peripheral input/output control lines; PB6, data bit of PIAI used to select channel; PTM, programmable timer module. 
controlled using a circulating water bath ( $t$ $=25 \pm 0.2^{\circ} \mathrm{C}$ ), while the surface pressure was measured in the right part. During the monolayer experiment, data (bar position) are stored every $10 \mathrm{~s}$ into the internal memory of the microprocessor. After the experiment data can be stored on diskettes for further analysis.

Surface pressure/molecular area curves. To interpret kinetic data obtained at constant surface pressure, it is essential to know the relationship between surface pressure and surface density, i.e., the number of molecules present per unit area. When surface pressure/ area curves were measured, the "zero-order trough" was replaced by the so-called "firstorder trough," which consists of only one compartment and has a rectangular form (13). The latter trough is larger than the first one, which enhances the measuring range, and its rectangular form simplifies molecular surface area calculations. Experiments were carried out with a known amount of lipid spread at the air/water interface ( 50 pmol di- $\mathrm{C}_{12} \mathrm{GS}$, initial pressure 0.07 dynes $/ \mathrm{cm}$ ). The film was compressed by moving the mobile barrier with a constant velocity of $9 \mathrm{~mm} / \mathrm{min}$. Surface pressure and bar position were monitored continuously on a Perkin-Elmer Model 56 recorder.

Apparatus. The monolayer system comprises a LM600 Microbalance which has analog output of the potential $(0-1 \mathrm{~V})$, a 10 turns precision potentiometer to give analog output of the position of the mobile barrier (0-5 V), and a Canon 2304 JJF DC motor to drive the barrier.

Hardware. The microprocessor used here is an Apple II computer (Apple Computer) with a 48-kilobyte RAM and a DISK II floppy disk subsystem (DOS 3.3) with two minifloppy disk drives with 116 kilobytes of storage capacity per disk. A Centronics printer (Data Computer) and Hewlett-Packard Model 7225A plotter provide communications and reporting facilities.

Programming language. The on-line control program and the off-line analyzing programs were written in Apple II BASIC (Applesoft).
The software routines for the interfaces were written in machine language (Assembler).

Interface design. In the computer-controlled monolayer system the microprocessor is interfaced to the microbalance, the DC motor, and the potentiometer (see Fig. 1). The system as depicted in Fig. 1 consists of three main parts: (a) a two-channel data-acquisition system (DAS), (b) a motor-control system, and (c) a programmable timer module (PTM). The data-acquisition system contains a two-channel 12-bit AD converter (Analog Devices, AD 574) which is coupled via 12 data input lines to a 16-bit peripheral interface adapter (Motorola, MC 6821) indicated as PIA1 in Fig. 1. The ADC (analog to digital converter) has a conversion time of $25 \mu \mathrm{s}$ per conversion. When a "read-peripheral data" command is given to the microprocessor, the CA2 peripheral output control line is set high as a signal for the AD converter to start data conversion. When data line "PB6" of PIAl is high, the analog output of the microbalance $(0-1 \mathrm{~V})$ is transferred via an amplifier (NE 5534, multiplication factor 5) to the ADC. When "PB6" is low, the analog output of the potentiometer is converted. The peripheral input line CAl of PIA1 is set after each conversion (EOC signal). Note that the analog output of the microbalance $(0-1 \mathrm{~V})$ corresponds with a pressure range of 0 to 25 dynes.

The motor-control system contains a peripheral interface adapter (PIA2) which is coupled via four data output lines to a simple DA converter. The CA2 control output line of PIA2 controls via a direction switch the direction of the DC motor. With CA2 high, $12 \mathrm{~V}$ is set over the motor and the barrier moves backward with a velocity of $10 \mathrm{~cm} /$ min. When the CA2 line is low, the DA converter is connected to the motor and the bar moves forward with a controllable velocity ( 9 steps from 9 to $40 \mathrm{~mm} / \mathrm{min}$ ). The barrier moves over a distance of $30 \mathrm{~cm}$. To protect the Wilhelmy plate against damages by themobile barrier, a microswitch is placed at a position of $29 \mathrm{~cm}$, which turns off the power of the motor when the bar reaches this po- 
sition. An identical switch is placed at a position of $0 \mathrm{~cm}$.

A programmable timer module (MC 6840) is introduced into the system to cause system interrupts at a constant time interval of $10 \mathrm{~s}$. An external crystal clock is connected to the PTM in order to generate a stable $1-\mathrm{kHz}$ input pulse.

On-line control program. Figure 2 shows a flow diagram of the on-line control program, MONOLAYER. The program uses three assembler subroutines (slave programs) which have the following functions. Program DAS initializes PIA1, disables the interrupt status, and converts the two channels (pressure and bar position) successively. In case of pressure reading the LSB (noise bit) of the 12 data bits is omitted, which gives rise to an accuracy of reading of 0.006 dynes (1/2 LSB). The bar position is read to an accuracy of $0.05 \mathrm{~mm}$ (12 bits). After data conversion the interrupt status is enabled. Program MOTOR initializes

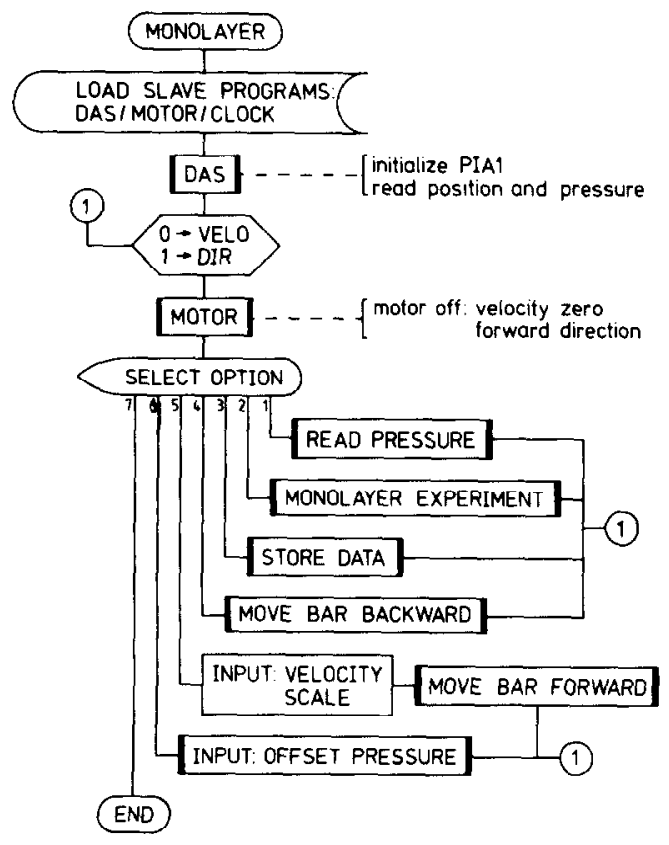

Fig. 2. Flow diagram of the on-line control program, MONOLAYER. The rectangular symbol with the two dark bars indicates a subroutine (BASIC) or a slave program (machine language).
PIA2 and reads the desired direction for bar movement (DIR). When DIR $=1$, the CA2 line is set low and a velocity value (VELO) is read. When DIR $=0$, the CA2 line is set high. Program CLOCK sets up the programmable timer module. A 16-bit counter is loaded with a decimal value of 10,000 , a value which is counted down with a $1-\mathrm{kHz}$ frequency supplied by the external crystal clock. When the counter value is zero (after $10 \mathrm{~s}$ ) an interrupt is generated, the counter is reloaded with its initial value, and the cycle is started again. Program CLOCK also sets up the interrupt routine in which the bar position is stored internally and the interrupt status enabled.

Program MONOLAYER comprises seven user-selectable options which cover all desired handling routines. Except for the above-mentioned three routines, all subroutines depicted in Fig. 2 are BASIC routines which are for the most part self-explanatory. The function of subroutine "MONOLAYER EXPERIMENT" is to keep pressure constant (tolerance $\pm 0.05 \mathrm{dynes} / \mathrm{cm}$ ) and to store the value of the bar position internally every $10 \mathrm{~s}$. After the experiment data can be stored on diskettes. The way of controlling bar movement in this subroutine needs further explanation. Determination of the bar velocity depends on the ratio $\left(\pi_{\mathrm{DES}}-\pi_{\mathrm{OBS}}\right) /$ proportional band, where $\pi_{\mathrm{DES}}$ is the desired pressure and $\pi_{\mathrm{OBS}}$ is the pressure observed. There exists an almost exponentional relationship between this ratio and the velocity. At high ratio values the velocity drop is much higher than at low ratios. The $\pi_{\text {DES }}$ and the proportional band are given as input to the "MONOLAYER EXPERIMENT" subroutine. The special function of the keyboard in the above kinetic program will be explained in the following section.

Off-line program "FILE SET UP." This program makes it possible to manipulate the obtained kinetic data in several ways before time-consuming calculations are carried out with the resulting data. (i) Any desired part of the curve can be discarded. This is especially needed when the curve bends off at high time values due to, e.g., absorption of the enzyme 
to the surface of the trough, enzyme inhibition by the products being formed, or denaturation of the enzyme molecule. (ii) The number of collected data can be reduced with a factor $n$ (an integer value) by increasing the time interval between two successive points. The new time interval must be a multiple $(n)$ of $10 \mathrm{~s}$. This option becomes important when measuring times get beyond $30 \mathrm{~min}$ ( 180 points). (iii) The curve can be split into $n$ parts with $n$ different time intervals, which is a simple way to give these parts different weighting factors in the subsequent analyzing program. (iv) A baseline can be subtracted from the titration curve. A mathematical equation for the baseline is derived from linear regression analysis.

The resulting data can be stored on diskette and retrieved by the following analyzing program.

Procedure of analysis. The analyses of the kinetic data were performed according to the method described by Verger et al. (14). A program was written in BASIC for nonlinear regression analysis based on the method of Fletcher and Powell (15). The unweighted kinetic data are fitted to Eq. [1], where $P$ is the bar position (mm) at time $t(\mathrm{~min}), v_{\mathrm{m}}$ the enzymatic velocity under steady-state conditions, and $\tau$ the induction time of the process,

$$
P=v_{\mathrm{m}} t+v_{\mathrm{m}} \tau\left(e^{-t / \tau}-1\right),
$$

which reflects penetration of the enzyme into the monolayer. From the above equation $v_{m}$ can be deduced to be the slope of the titration curve when time approaches to infinity, i.e., the asymptotic value $(t \gg \tau)$. Extrapolation of this asymptote to the $x$ axis gives the value for $\tau$.

In the model as described by Verger $e t$ al. (14) it is assumed that the induction time of the penetration process is much longer than the induction time which describes the establishment of the interfacial Michaelis-Menten equilibrium. It was also deduced that $v_{\mathrm{m}}$ should be proportional to total enzyme concentration.

\section{RESULTS AND DISCUSSION}

\section{Test and Calibration of Monolayer System}

Test experiments revealed that optimal use is made of the computer-controlled monolayer system when the following handling conditions are met. (i) After the trough is filled with buffer and the surface is cleaned, the pressure is read with user-selectable option 1 of program MONOLAYER. (ii) When pressure becomes constant, this value is determined to be the offset value for all successive pressure readings: option 6. (iii) The lipid film is spread to a pressure somewhat below the desired one. (iv) The film is brought to the desired pressure ( $\left.\pi_{\text {DES }}\right)$ and held constant with option 2. (v) When the baseline becomes linear, key " $\mathrm{A}$ " is pressed to start data accumulation. On the pressing of any other key, the process is stopped and the baseline data can be stored on diskette. (vi) Option 2 is used again for restoring the baseline. Enzyme is injected under the film in the left compartment, key " $A$ " is pressed, and the kinetic data are collected. On the pressing of any other key, data can be stored on diskette and retrieved again in the data manipulation and analyzing programs.

The exponential relationship between the velocity of the DC motor and the ratio ( $\pi_{D E S}$ - $\pi_{\mathrm{OBS}} /$ /proportional band gives rise to an optimum value for the proportional band, which can be different for each substrate used. When the band is too small, a small decrease in $\pi_{\mathrm{OBS}}$ relative to $\pi_{\mathrm{DES}}$ will result in too high a compression velocity. Equilibrium conditions cannot be reached and $\pi_{\mathrm{OBS}}$ will pass $\pi_{\text {DES }}$. The kinetic curve will not have a smooth continuous form but a stepped one. If the proportional band is too large, there is a high risk that $\pi_{\text {DES }}$ will never be reached. An optimum value of 0.02-0.05 dynes was used for the studied substrate films of di- $\mathrm{C}_{12} \mathrm{GS}$ and di$\mathrm{C}_{10} \mathrm{PC}$.

One way to test a new experimental setup is to try to reproduce previously obtained results. Degradation of a monomolecular film of di- $\mathrm{C}_{10} \mathrm{PC}$ by increasing amounts of phos- 

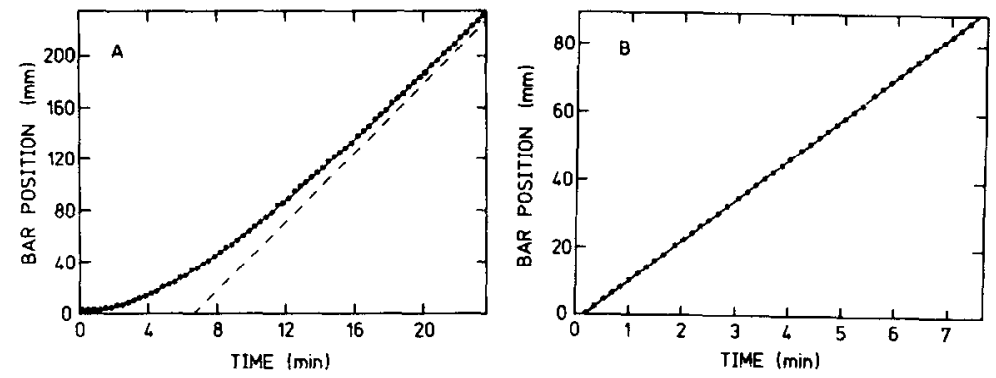

FIG. 3. (A) Kinetics of the hydrolysis of a 1,2-didodecanoyl-sn-glycero-3-sulfate film upon injection of $10 \mu \mathrm{g}$ of human pancreatic phospholipase $A_{2}$. Surface pressure 13 dynes $/ \mathrm{cm}$. (B) Hydrolysis of a 1,2didecanoyl-sn-glycero-3-phosphocholine upon injection of $7 \mu \mathrm{g}$ of bee venom phospholipase $A_{2}$. Surface pressure 5 dynes/cm. Experimental conditions: Tris buffer, $10 \mathrm{mM}, \mathrm{pH} 8.0 ; \mathrm{NaCl}, 100 \mathrm{mM} ; \mathrm{CaCl}_{2}, 20 \mathrm{~mm}$. $T=25^{\circ} \mathrm{C}$. The continuous curves represent the results of the computer fit. The points on the continuous curves represent data points being collected in program MONOLAYER. Curve (B) shows all collected data (time interval $10 \mathrm{~s}$ ), whereas curve (A) shows the data at a time interval of $20 \mathrm{~s}$. The dashed line in (A) is the computed asymptote of the curve. In (B), the asymptote and the computed titration curve are overlapping.

pholipase $\mathrm{A}_{2}$ from pig pancreas at a pressure of 11.5 dynes $/ \mathrm{cm}, \mathrm{pH} 8,20 \mathrm{mM} \mathrm{CaCl}_{2}$, demonstrated a linear relationship between the amounts of total enzyme injected (1-10 $\mu \mathrm{g})$ and the hydrolysis rate $v_{\mathrm{m}}$ (expressed in $\mathrm{mm} /$ min). Calculation of $v_{m}$ in more standardized units gave values of about 9 lipid molecules/ $\mathrm{cm}^{2} / \mathrm{min} / 10 \mu \mathrm{g}$ PLA injected or $1.5 \mu \mathrm{eq} / \mathrm{min} /$ mg PLA injected. The induction time $\tau$, being approximately $8 \mathrm{~min}$, was independent of the amount of enzyme injected. These results agree satisfactorily with those obtained by Verger and de Haas (13) and Verger et al. (14). Efficient stirring of the left compartment was tested by hydrolysis of a lipid film of di$\mathrm{C}_{10} \mathrm{PC}$ by PLA from bee venom $(7.5 \mu \mathrm{g})$, at a pressure of 5 dynes/cm, pH 8, $20 \mathrm{mM} \mathrm{CaCl}_{2}$ (see Fig. 3). This enzyme gave linear kinetics from the first few seconds onward as was demonstrated previously (14).

\section{Application to Phospholipases $A_{2}$}

Figure 4 shows the apparent activity profiles for phospholipases $A_{2}$ from various pancreatic sources as a function of surface pressure. Figure 5 represents the corresponding lag time profiles. The velocity values are apparent specific activities because the amount of enzyme adsorbed to the interface is not known. As

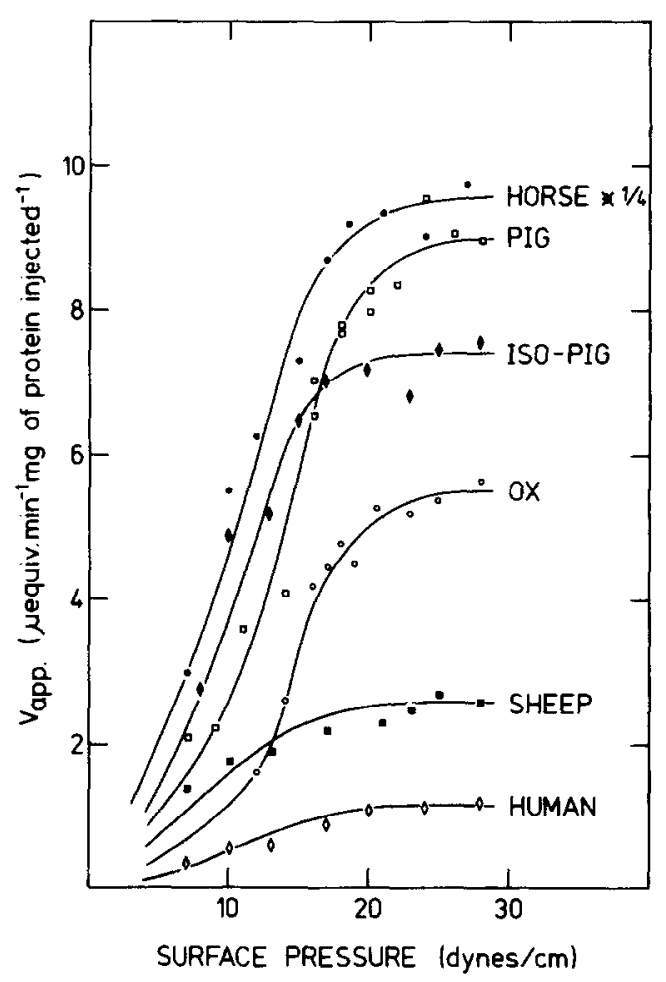

FiG. 4. Influence of surface pressure on the apparent velocity of various PLAs acting on di- $\mathrm{C}_{12} \mathrm{GS}$. Standard buffer, $\mathrm{pH} 8, T=25^{\circ} \mathrm{C}$. Amount of enzyme injected: 1$10 \mu \mathrm{g}$. PLA from $(\diamond)$ man, $(0)$ sheep, $(0)$ ox, $(\diamond)$ pig (isoenzyme), ( $\square$ ) pig, and (0) horse. Note: the apparent velocity values for the equine enzyme were divided by a factor of 4. 


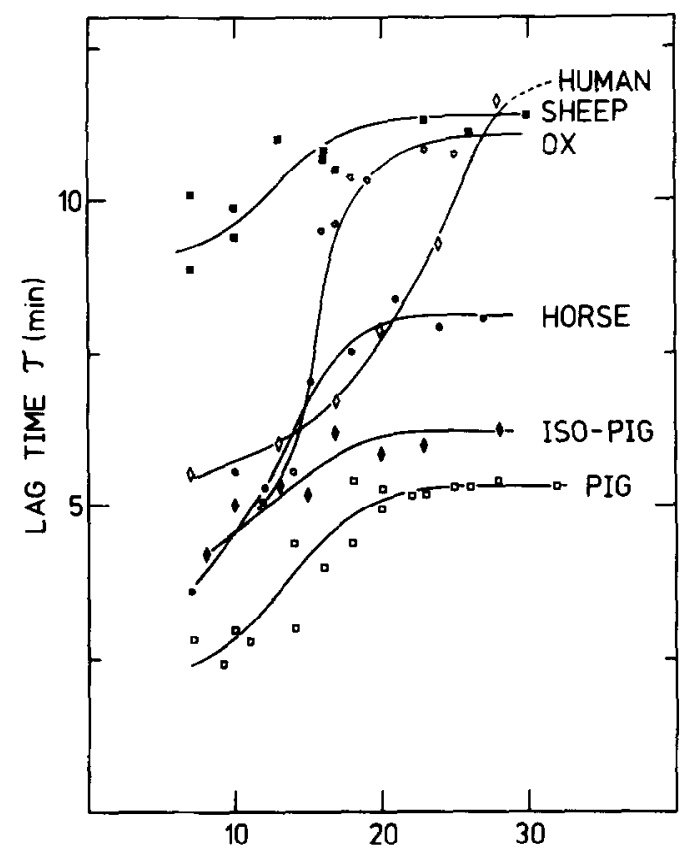

SURFACE PRESSURE (dynes/cm)

FiG. 5. Influence of surface pressure on the lag time of various PLAs acting on di- $\mathrm{C}_{12}$ GS. Same conditions as given in Fig. 4. PLA from $(\diamond)$ man, $(D)$ sheep, $(O)$ ox, $(\diamond)$ pig (isoenzyme), ( $\square$ ) pig, and (๑) horse.

shown by Pattus et al. (16), only a very small amount of the enzyme injected will be present in the monolayer. The apparent velocity values expressed in units of equivalents/minute/milligram enzyme injected could be calculated after determination of the relationship surface pressure/area per molecule in the monolayer system (option 5 of program MONOLAYER). The collapse pressure was determined to be 36-39 dynes/cm for the lipid di- $\mathrm{C}_{12} \mathrm{GS}$ (pH $8,20 \mathrm{mM} \mathrm{CaCl}_{2}$ ) with a corresponding area/ molecule of $50 \AA^{2}$. In the absence of $\mathrm{Ca}^{2+}$ the area per molecule increased to a value of 55 $\AA^{2}$. Velocity measurements were not performed at surface pressures above $\mathbf{3 0}$ dynes/ $\mathrm{cm}$ due to an increasing instability of the lipid film above this pressure.

The observed differences between the various studied PLAs can be related to several aspects of substrate binding and its chemical conversion and will be discussed elsewhere. Apart from hydrophobic binding $(17,18)$ there is also evidence for polar interaction with negatively charged groups in the surface (19-21). From a comparison of Fig. 4 with Fig. 5 at a pressure of about 25 dynes $/ \mathrm{cm}$ (maximum of velocity profiles), it can be seen that a gradual increase of penetration capacity in the series of studied PLAs (excluding the equine enzyme) coincides with a gradual increase in enzymatic velocities. It is highly tentative to assume that due to increasing polar interactions in the series of PLAs, more enzyme gets adsorbed to the interface resulting in higher apparent hydrolysis rates.

The interfacial regulation of PLA activity on these negatively charged surface films is under investigation by using radioactively labeled enzymes to determine the amount of enzyme present in the monolayer.

\section{REFERENCES}

1. van Deenen, L. L. M., and de Haas, G. H. (1963) Biochim. Biophys. Acta 70, 538-553.

2. Bonsen, P. P. M., de Haas, G. H., Pieterson, W. A., and van Deenen, L. L. M. (1972) Biochim. Biophys. Acta 270, 364-382.

3. Nieuwenhuizen, W., Kunze, H., and de Haas, G. H. (1974) in Methods in Enzymology (Fleischer, S., and Packer, L., eds.), Vol. 32 Part B, pp. 147-154, Academic Press, New York.

4. Fleer, E. A. M., Verheij, H. M., and de Haas, G. H. (1978) Eur. J. Biochem. 82, 261-270.

5. Dutilh, C. E., van Doren, P. J., Verheul, F. E. A. M., and de Haas, G. H. (1975) Eur. J. Biochem. 53, 91-97.

6. Evenberg, A., Meijer, H., Verheij, H. M., and de Haas, G. H. (1977) Biochim. Biophys. Acta 491, 265274.

7. Grataroli, R., de Caro, A., Guy, O., and Figarella, C. (1981) Biochimie 63, 677-684.

8. Grataroli, R., Dijkman, R., Dutilh, C. E., van der Ouderaa, F., de Haas, G. H., and Figarella, C. (1982) Eur. J. Biochem. 122, 111-117.

9. Shipolini, R. A., Collewaert, G. L., Cottrell, R. E., Doonan, S., and Vernon, C. A. (1971) Eur. J. Biochem. 20, 459-468.

10. Hille, J. D. R. (1983) Ph.D. Thesis, State Univer. of Utrecht, Utrecht, The Netherlands.

11. Gupta, C. M., Radhakrishnan, R., and Khorana, 
H. G. (1977) Proc. Natl. Acad. Sci. USA 74, 43154319.

12. Cubero Robles, E., and van den Berg, D. (1969) Biochim. Biophys. Acta 187, 520-526.

13. Verger, R., and de Haas, G. H. (1973) Chem. Phys. Lipids 10, 127-136.

14. Verger, R., van Dam-Mieras, M. C. E., and de Haas, G. H. (1973) J. Biol. Chem. 248, 4023-4034.

15. Fletcher, R., and Powell, M. J. D. (1963) Comput. J. 6, 163-173.

16. Pattus, F., Slotboom, A. J., and de Haas, G. H. (1979) Biochemistry 13, 2691-2697.
17. de Araujo, P. S., Rosseneu, M. Y., Kremer, J. M. H., van Zoelen, E. J. J. and de Haas, G. H. (1979) Biochemistry 18, 580-586.

18. Donné-Op den Kelder, G. M., Hille, J. D. R., de Haas, G. H., and Egmond, M. R. (1981) Biochemistry 20, 4074-4078.

19. Wells, M. A. (1974) Biochemistry 13, 2265-2268.

20. Willman, C., and Hendrickson, H. S. (1978) Arch. Biochem. Biophys. 191, 298-305.

21. Hille, J. D. R., Egmond, M. R., Dijkman, R., van Oort, M. G., Sauve, P., and de Haas, G. H. (1983) Biochemistry 22, 5358-5364. 